\title{
BMJ Open Patient-reported outcomes at hospital discharge from Heart Centres, a national cross-sectional survey with a register- based follow-up: the DenHeart study protocol
}

\author{
Selina Kikkenborg Berg, ${ }^{1,2}$ Jette Svanholm, ${ }^{3}$ Astrid Lauberg, ${ }^{4}$ Britt Borregaard, ${ }^{5}$ \\ Margrethe Herning, ${ }^{2}$ Anna Mygind, ${ }^{3}$ Anne Vinggaard Christensen, ${ }^{1}$ \\ Anne Illemann Christensen, ${ }^{6}$ Ola Ekholm, ${ }^{6}$ Knud Juel, ${ }^{6}$ Lars Thrysøe ${ }^{7}$
}

To cite: Berg SK, Svanholm J, Lauberg A, et al. Patientreported outcomes at hospital discharge from Heart Centres, a national crosssectional survey with a register-based follow-up: the DenHeart study protocol. BMJ Open 2014;4:e004709. doi:10.1136/bmjopen-2013004709

- Prepublication history for this paper is available online. To view these files please visit the journal online (http://dx.doi.org/10.1136/ bmjopen-2013-004709).

Received 18 December 2013 Revised 9 April 2014 Accepted 11 April 2014

\section{CrossMark}

For numbered affiliations see end of article.

Correspondence to Dr Selina Kikkenborg Berg; selina@rh.dk

\section{ABSTRACT}

Introduction: Patient reported health status, which includes symptom burden, functional status and quality of life, is an important measure of health. Differences in health status between diagnostic groups within cardiology have only been sparsely investigated. These outcomes may predict morbidity, mortality, labour market affiliation and healthcare utilisation in various diagnostic groups. A national survey aiming to include all cardiac diagnostic groups from a total Heart Centre population has been designed as the DenHeart survey.

Methods and analysis: DenHeart is designed as a cross-sectional survey with a register-based follow-up. All diagnostic groups at the five national Heart Centres are included during 1 year (15 April 2013 to 15 April 2014) and asked to fill out a questionnaire at hospital discharge. The total eligible population, both responders and non-responders, will be followed in national registers. The following instruments are used: SF-12, Hospital Anxiety and Depression Scale, EQ-5D, Brief Illness Perception Questionnaire (B-IPQ), HeartQoL and Edmonton Symptom Assessment Scale. The following variables are collected from national registers: action diagnosis, procedures, comorbidity, length of hospital stay, type of hospitalisation, visits to general practitioners and other agents in primary healthcare, dispensed prescription medication, vital status and cause of death. Labour market affiliation, sick leave, early retirement pension, educational degree and income will be collected from registers. Frequency distributions and multiple logistic regression analyses will be used to describe and assess differences in patient reported outcomes at hospital discharge between diagnostic groups and in-hospital predicting factors. Cox proportional hazards regression models with age as the time scale will be used to investigate associations between patient reported outcomes at baseline and morbidity/mortality, labour market affiliation and healthcare utilisation after 1 year.

Ethics and dissemination: The study complies with the Declaration of Helsinki. The study has been approved by the Danish Data Protection Agency: 200758-0015/30-0937 and registered at ClinicalTrials.gov (NCT01926145). Study findings will be disseminated widely through peer reviewed publications and conference presentations.

\section{INTRODUCTION}

Patient reported health status, which includes symptom burden, functional status and quality of life (QoL), is an important measure of health. Validated patient health status surveys, including disease-specific instruments for patients with cardiovascular disease, allow quantification of critical patient-centred outcomes and additional research is needed to better understand the determinants ${ }^{1}$ and the predicting factors of patients' health status. Previous studies suggest an association between heart disease, self-reported health and morbidity and mortality, and that patient reported outcome measures can predict prolonged hospital stay, future QoL, return to work, and morbidity and mortality in cardiac patients. ${ }^{2-7}$ QoL scores seem to provide important prognostic information independent of traditional clinical data, as higher scores have been associated with longer survival in patients with ventricular arrhythmias and coronary artery disease. ${ }^{8}{ }^{9}$ However, no studies have included all diagnostic groups within cardiology and comparisons among diagnostic groups are lacking. The overall aim of the DenHeart survey is to gain knowledge about patient reported outcome measures regarding health among cardiac patients at hospital discharge. Knowledge about patients' own 
perception of their health status and predicting factors can help to guide inpatient practice and outpatient follow-up. Furthermore, a survey combined with register data can be used to evaluate differences among diagnostic groups and predicting factors for patient-reported outcome measures at hospital discharge and long-term morbidity and mortality. Also, economic analysis of healthcare utilisation and work ability status in a large cohort of cardiac patients is needed. Therefore, the DenHeart study is designed as a national survey aiming to include all cardiac diagnostic groups from a total Heart Centre population.

\section{OBJECTIVES}

The objectives of the DenHeart study are to describe: (1) differences in patient-reported outcomes at hospital discharge between diagnostic groups and in-hospital predicting factors and (2) patient-reported outcomes at hospital discharge as a predictor of (a) morbidity and mortality, (b) labour market affiliation and (c) healthcare utilisation after 1 year.

\section{METHODS AND ANALYSIS}

\section{Study design}

The DenHeart study is designed as a cross-sectional survey with a register-based follow-up. All cardiac patients are asked to fill out a questionnaire at hospital discharge to evaluate patient-reported outcomes. Furthermore, the total eligible population, responders and non-responders, will be followed in national registers.

\section{Setting and participants}

The five Heart Centres in Denmark are including patients during a 1-year period, from 15 April 2013 to 15 April 2014. One centre began data collection later, 1 May 2013. Four heart centres have both medical and surgical wards, and one centre only has a medical ward. All cardiac patients discharged or transferred to a local hospital from one of the Heart Centres are potential participants in the study. Patients are unselected and consecutively included at hospital discharge. Included patients are asked to complete and return a questionnaire before they leave the hospital or, alternatively, to do so at home within 3 days of discharge and return it by mail. Patients who are transferred to another hospital are given the questionnaire at discharge from the Heart Centre and asked to fill it out on the day of hospital discharge or, alternatively, to do so at home within 3 days of discharge and then return it by mail.

\section{Eligibility criteria}

All diagnostic groups within cardiology are included. Patients with ischaemic heart disease (eg, coronary angiography, percutaneous coronary intervention), heart failure (eg, heart transplantation), arrhythmia (eg, ablation, labyrinth, pacemaker, implantable cardioverter defibrillator), heart valve disease (eg, valve replacement), endocarditis and congenital heart disease (eg, atrium septum defect, patent ductus arteriosus, patent foramen ovale, coarctatio) are diagnosed and treated at the Heart Centres. Infrequent conditions such as thorax trauma are also included. Patients are grouped by their primary action diagnosis and will only be included in one group.

Patients under 18 years of age and patients without a Danish civil registration number are excluded from the study. For ethical reasons, patients who are unconscious when transferred are also excluded.

Reasons for non-response are recorded which allows for subanalyses of these groups. On the front page of the questionnaire, there is a box that enables the patient or nurse to tick off the reason for non-response: 'Does not wish to participate', 'Not able to participate because of illness', 'Not able to participate because of language barrier', 'Questionnaire not handed out' and 'Other'.

\section{Recruitment}

Patients are recruited at hospital discharge (on the same day or the day before discharge) by the ward nurse in charge of the discharge of the individual patients or by a research nurse. All nurses at the centres, approximately 800 , have been informed about the study and procedures at ward meetings; guidelines have been distributed and a website created (http://www.DenHeart.dk). When informing patients about the study and handing out the questionnaire, nurses also distribute a postage prepaid envelope for the return of the questionnaire, either at the ward or after discharge. No reminders are sent to patients as the time window of 3 days postdischarge makes it impossible. Distribution and return rates are monitored to allow for interventions if the rates drop during data collection. No specific cut-off is set for low rates calling for interventions. Instead, monthly discussions on each site and in the national research group are undertaken, allowing for discussions and ideas for reminding the staff to hand out to the questioners.

\section{Data sources/measurement}

The following patient-reported outcome measures are used: Short-form 12 (SF-12), Hospital Anxiety and Depression Scale (HADS), EQ-5D, Brief Illness Perception Questionnaire (B-IPQ), HeartQoL and Edmonton Symptom Assessment Scale (ESAS). The EQ5D and SF-12 are generic health instruments. The SF-12 is included to be able to compare to a national general population and the EQ-5D is included due to a different scale composition.

SF-12: The SF-12 is a 12-item version of the SF-36 and is a brief, reliable measure of overall health. The questionnaire measures eight domains of health: physical function, role-physical, bodily pain, general health, vitality, social functioning, role-emotional and mental health with higher scores indicating better health status. The items cover the previous 4 weeks and the results are 
expressed in terms of two summary scores: the Physical Component Summary (PCS) and the Mental Component Summary (MCS).${ }^{10}$ Cronbach's $\alpha$ values of 0.87 and 0.84 for PCS-12 and MCS-12, respectively, have been reported in a population of coronary heart disease patients. ${ }^{11}$ The SF-12 is suitable for studies focusing on patient-based assessment of physical and mental health. ${ }^{10}$ Furthermore, it is used in the National Health Surveys and outcomes can be compared.

HADS: The HADS is a 14-item questionnaire that assesses levels of depression and anxiety in medically ill patients admitted to non-psychiatric hospital clinics. The scale offers two scores, HADS-A and HADS-D, and consists of seven questions to assess anxiety and seven questions to assess depression. ${ }^{12}$ For each of the questions, the respondent chooses from four responses to indicate the extent to which each applies to the previous week. The HADS is a valid and internally consistent measure, with a mean Cronbach's $\alpha$ of 0.83 and 0.82 for the HADS-A and HADS-D, respectively. ${ }^{13}$ Scores of $0-7$ for either subscale are regarded as normal and scores of 810 suggest the presence of a mood disorder. Scores of 11 and above indicate the probable presence of a mood disorder. ${ }^{14}$

$E Q-5 D$ : The EQ-5D is a six-item standardised instrument for measuring current health status that provides a simple descriptive profile and a single index value that can be used in the clinical and economic evaluation of healthcare and in population health surveys. The questionnaire covers five dimensions of health: mobility, selfcare, usual activities, pain/discomfort and anxiety/ depression. Each dimension is divided into three levels: no problems, some or moderate problems or extreme problems. The sixth item, a Visual Analogue Scale, enables the respondent to provide a self-rating of his/ her own health. Higher scores indicate a better health status. ${ }^{15}$ An overall Cronbach's $\alpha$ of 0.73 has been found in a population of coronary heart disease patients. ${ }^{11}$

$B$-IPQ: The B-IPQ is a short eight-item questionnaire that assesses cognitive and emotional representations of illness. Each of the eight items represents a dimension of the respondent's perception of his or her own illness. Five items assess current cognitive representations of illness: consequences, timeline, personal control, treatment control and identity. Two items assess emotional representations of illness: concern and emotions. The last item assesses illness comprehensibility. A higher score on the B-IPQ reflects a more threatening view of illness. The B-IPQ has good test-retest reliability measured with the Pearson correlations and has shown good predictive validity among patients recovering from myocardial infarction. ${ }^{16}$

HeartQoL: The HeartQoL is a 14 -item illness-specific questionnaire that measures the QoL in cardiac patients. The questionnaire covers the previous 4 weeks and produces a global score and two subscales. A physical and an emotional scale with higher scores indicate a better QoL status. The HeartQoL is a new questionnaire developed on the basis of items from three widely used questionnaires for specific groups of cardiac patients (the Seattle Angina Questionnaire, the MacNew Heart Disease Health-related Quality of Life Questionnaire and the Minnesota Living with Heart Failure Questionnaire). The questionnaire has proven to be a reliable instrument with a Cronbach's $\alpha$ between 0.80 and 0.91 for the global score and each subscale and to be responsive in patients with a wide spectrum of diagnoses. ${ }^{17-19}$

ESAS: The ESAS is a 10-item questionnaire that allows patients to rate their current symptoms on a visual numeric scale. The following symptoms are included: pain, tiredness, nausea, depression, anxiety, drowsiness, appetite, well-being, shortness of breath and distress. Higher scores indicate the presence and intensity of the symptoms. The ESAS has proven to be a valid instrument to measure the self-reported symptoms of patients with cancer with an overall Cronbach's $\alpha$ of $0.79 .{ }^{20}$ Even though the ESAS was developed for palliative care in patients with cancer, it has been used in cardiac populations and found to have modest correlation to the New York Heart Association class and heart failure questionnaires. ${ }^{21}$ Besides the validated questionnaires, 16 questions about health behaviour, cardiac symptoms, sense of security and use of medicines are included in the questionnaire. This amounts to a total of 80 items. The questionnaire was pretested for feasibility by $12 \quad(10$ men, 2 women) patients aged 52-81-years (mean 65.9) on medical and surgical wards at three of the Heart Centres, and the introduction and layout was adjusted afterwards. The questionnaire takes about $20 \mathrm{~min}$ to complete.

\section{Variables from registers}

All Danish citizens have a unique personal identification number; linkage between the national registers and other data sources is feasible. Therefore, the Danish registers offer a great number of possibilities for national epidemiological studies. ${ }^{22}$

For the DenHeart study, data are drawn at baseline and follow-up after 1 year from the following registers:

The Danish Civil Registration System ${ }^{23}$ : Gender, age, marital status.

The Danish National Patient Register ${ }^{24}$ : Action diagnosis, other diagnoses, procedures, length of hospital stay, type of hospitalisation (acute, heart related, other).

The Danish National Health Service Register ${ }^{25}$ : Contact with general practitioners and other agents in primary healthcare.

Registers on personal labour market affiliation ${ }^{26}$ : Labour market affiliation, sick leave, early retirement pension.

Population Education Register ${ }^{27}$ : Educational degree. The Income Statistics Register ${ }^{28}$ : Income.

\section{Data handling and record keeping}

Questionnaires are mailed from the Heart Centres or from the patients' home to a scanning agency (Express 
A/S). Questionnaires are scanned and the file delivered to The National Institute of Public Health, University of Southern Denmark and placed on a secure hard drive. When the data collection is finished, the entire dataset will be reviewed in order to remove ineligible respondents, for example, pulmonary trauma. Furthermore, the entire population of patients discharged from the Heart Centres during the data collection period will be drawn from the National Patient Register. This will be reviewed in order to remove irrelevant discharges, in accordance with the inclusion criteria.

\section{Data monitoring}

In order to monitor trial conduct, reports are continuously delivered from the scanning agency to identify the need for adjustments. A data file is delivered every 2 weeks during the first 3 months and every month thereafter throughout the entire data collection period. The data file includes a list of received questionnaires ranked by centre and unit, and information on whether the questionnaire has been completed on the front page. The numbers are compared to the number of patients discharged at unit level in the same period. Assessments of response rates are performed and adjustments made if needed. Low response rates in a unit will lead to contact with the unit concerned to learn the reason why and actions to increase them. Returned questionnaires will be screened for systematic errors.

\section{Study size}

The study size is not derived statistically, as it consists of the total population of patients discharged from the five Heart Centres in the project period. The five Heart Centres diagnose and treat about 45000 patients per year. These patients are diagnosed with numerous diseases and conditions and the aim is to describe differences not only among the most common diagnoses but also among the infrequent ones. Thus, all patients discharged from national Heart Centres over 1 year are included in order to secure as high a level of specificity as possible. Furthermore, by including a large number of participants, a shorter follow-up time is needed. Besides the primary objectives, further ancillary and spin-off analyses will be prepared. Clearly, power calculations cannot be performed prospectively for all potential analyses based on data from the DenHeart study. Moreover, adverse outcomes have very different incidence rates.

\section{Response rate}

The final response rate is calculated based on discharge data from the National Patient Register. For calculating the actual number of eligible patients, the following are not included: patients without a Danish civil registration number, patients living in Greenland (no register follow-up is possible), patients with two admissions within $24 \mathrm{~h}$, patients deceased in hospital and outpatient visits. Patients admitted for less than $24 \mathrm{~h}$ without a procedure and readmitted with a procedure within 1 month are counted once.

Even though patients are encouraged to fill out the questionnaire within 3 days, all questionnaires completed within 4 weeks of discharge are included in the analyses.

Patients who have filled out more than one questionnaire during the project period due to repeated hospitalisations will only be counted once (when the first questionnaire is returned) in the final count of the response rate. The repeated measurements may be included in subanalyses.

\section{Statistical methods}

Objective 1: For each diagnostic group, age (mean), sex distribution and patient-reported outcomes at hospital discharge distribution will be reported. Differences between groups will be tested by use of analysis of variance (ANOVA) tests for continuous variables and $\chi^{2}$ tests for categorical variables. Multivariate logistic regression will be used to investigate the relationship between the diagnostic group and patient-reported outcomes when controlling for response date (number of days since hospital discharge), age, sex and other possible confounders and in-hospital predicting factors (eg, procedure, duration of index hospital admission, acute/elective admission, stay at intensive care unit, complications).

Objective 2a: For each diagnostic group, the crude associations between patient-reported outcome measures at hospital discharge and all-cause and cardiac morbidity and all-cause and cardiac mortality after 1 year will be described and assessed using ANOVA tests for continuous variables and $\chi^{2}$ tests for categorical variables. In addition, multivariate Cox proportional hazards regression models, with age as the time scale, will be used to examine the associations between patient-reported outcome measures at baseline and all-cause morbidity and mortality and cardiac morbidity and mortality when adjusting for response date, sex, comorbidity and other potential confounding factors at baseline.

Objective 2b: For each diagnostic group, the crude associations between patient-reported outcome measures at hospital discharge and labour market affiliation (employment status, sick leave, early retirement pension) after 1 year will be described and assessed using ANOVA tests for continuous variables and $\chi^{2}$ tests for categorical variables. Multivariate Cox proportional hazards regression models, with age as the time scale, will also be carried out to investigate the associations between patient-reported outcome measures at baseline and labour market affiliation when adjusting for potential confounding factors (eg, response date, sex, comorbidity).

Objective 2c: For each diagnostic group, the crude associations between patient-reported outcome measures at hospital discharge and healthcare utilisation (hospitalisations, contact with general practitioner, medical specialist and physiotherapist) after 1 year will be described 
and assessed using ANOVA tests for continuous variables and $\chi^{2}$ tests for categorical variables. Multivariate Cox proportional hazards regression models, with age as the time scale, will be used to analyse the association between patient-reported outcome measures at baseline and healthcare utilisation when adjusting for potential confounding variables (eg, response date, sex, comorbidity).

\section{Weighting}

It is possible to link both respondents and non-respondents in the survey on an individual level to different administrative registers. Thus, non-response weights will be computed based on register information (eg, sex and age) in order to reduce non-response bias. Patients with a low probability of response will be given a higher weight in the analyses to represent the larger number of non-respondents with similar characteristics. Accordingly, patients more likely to respond will be given a lower weight.

\section{Spin-off projects}

Several spin-off analyses will be prepared.

\section{Ethics and dissemination}

The study is conducted in accordance with the Declaration of Helsinki. According to Danish legislation, surveys should not be approved by an ethics committee system but only by the Danish Data Protection Agency, no. 2007-58-0015/30-0937. The DenHeart survey is approved by the Institutional Boards of the Heart Centres and registered at ClinicalTrials.gov (NCT01926145). Patients sign informed consent stating that participation is voluntary and that further information from patient records may be obtained.

The National Institute of Public Health stores data from the DenHeart survey. Access to data can only be provided to investigators after approval from the Publication Committee. All study results will be published in international research journals and conference presentations.

\section{DISCUSSION}

\section{Study population}

The five Heart Centres treat the most critically ill cardiac patients in Denmark. Approximately 47 regional or local hospitals treat cardiac patients medically and care for terminally ill cardiac patients. These patients are underrepresented in the DenHeart study. The patients in this study represent patients with diagnoses where medical, invasive or surgical treatment is possible, and patients with rare conditions such as heart transplant and congenital heart disease. Furthermore, four of the five Heart Centres have a local hospital function treating all cardiac diagnoses. One surgical unit did not wish to participate in the study.
Patients who are hospitalised several times in the project period will be asked to fill out the questionnaire each time. Repeated measures will only be used in subanalyses. For reporting of primary outcomes, patients will only be counted once (when the first questionnaire is returned).

\section{Variables}

The majority of the outcome measures included in the questionnaire is comprised of validated and standardised instruments to assess patient-reported outcomes, which enhances the validity of the questionnaire. However, in the interpretation of the results, it must be taken into account that patients with different cardiac conditions differ regarding how much their health status has changed prior to admission to hospital and that some of the instruments have long recall.

\section{Confounding factors}

From previous national health surveys, we know that age and socioeconomic position play a significant role in nonresponse. Also, regional differences of about $15 \%$ in response rates have been documented. ${ }^{29}{ }^{30}$ The patients treated at the Heart Centres are often aged and severely ill, which may be reflected in the response rates. This will be accounted for by reporting the actual responses as well as the weighted data for the under-represented groups in the analyses. However, several different initiatives are being conducted by the centres to optimise response rates. Nurses are reminded to hand out the questionnaires at local meetings, by posters in the ward and logo-pens and response rates are continuously shared with the ward nurses in order to secure motivation among the approximately 800 nurses involved in the distribution of questionnaires. Letters are sent to the local hospitals that receive patients from the Heart Centres asking them to remind the patients to fill out the questionnaire on discharge to their home. Some centres are mailing questionnaires to patients after discharge when they are not handed out in hospital. Mailed questionnaires are sent with overnight postservice allowing the questionnaire to be filled out within 3 days. As this is a national multicentre study, regional differences in ways to enhance the response rates may occur. All of these different strategies fall within the frame of the DenHeart study and timeframe for responding.

\footnotetext{
Author affiliations

${ }^{1}$ The Heart Centre, Unit 2151, Rigshospitalet, Copenhagen University Hospital, Copenhagen, Denmark

${ }^{2}$ Department of Cardiology, Gentofte University Hospital, Hellerup, Denmark ${ }^{3}$ Department of Cardiology, Aarhus University Hospital, Aarhus N, Denmark

${ }^{4}$ Departments of Cardiology and Cardiothoracic Surgery, Aalborg University Hospital, Aalborg, Denmark

${ }^{5}$ Department of Thoracic, Cardiac and Vascular Surgery, Odense University Hospital, University of Southern Denmark, Odense C, Denmark

${ }^{6}$ National Institute of Public Health, University of Southern Denmark, Copenhagen, Denmark

${ }^{7}$ Department of Cardiology, Odense University Hospital, University of Southern Denmark, Odense C, Denmark
} 
Contributors SKB conceived the idea of the study. SKB, AVC, MH, LT, BB, JS, $A M, A L, A I C, K J$ and $O E$ initiated the study design and implementation. $\mathrm{KJ}, \mathrm{AIC}$ and $\mathrm{OE}$ provided statistical expertise in the clinical trial design. All contributed to the refinement of the study protocol.

Funding This work was supported by the five participating Heart Centres in Denmark. Further funding will be applied for from external sources.

\section{Competing interests None.}

Patient consent Obtained.

Provenance and peer review Not commissioned; externally peer reviewed.

Open Access This is an Open Access article distributed in accordance with the Creative Commons Attribution Non Commercial (CC BY-NC 3.0) license, which permits others to distribute, remix, adapt, build upon this work noncommercially, and license their derivative works on different terms, provided the original work is properly cited and the use is non-commercial. See: http:// creativecommons.org/licenses/by-nc/3.0/

\section{REFERENCES}

1. Rumsfeld JS, Alexander KP, Goff DC, et al. Cardiovascular health the importance of measuring patient-reported health status: a scientific statement from the American Heart Association. Circulation 2013;127:2233-49.

2. Pressler SJ, Kim J, Riley P, et al. Memory dysfunction, psychomotor slowing, and decreased executive function predict mortality in patients with heart failure and low ejection fraction. J Card Fail 2010;16:750-60.

3. Hoekstra T, Jaarsma T, van Veldhuisen DJ, et al. Quality of life and survival in patients with heart failure. Eur J Heart Fail 2013;15:94-102.

4. Soto GE, Jones P, Weintraub WS, et al. Prognostic value of health status in patients with heart failure after acute myocardial infarction. Circulation 2004;110:546-51.

5. Heidenreich PA, Spertus JA, Jones PG, et al. Health status identifies heart failure outpatients at risk for hospitalization or death. J Am Coll Cardiol 2006;47:752-6.

6. Biering K, Nielsen TT, Rasmussen K, et al. Return to work after percutaneous coronary intervention: the predictive value of self-reported health compared to clinical measures. PLoS ONE 2012;7:e49268.

7. Szekely A, Nussmeier NA, Miao Y, et al. A multinational study of the influence of health-related quality of life on in-hospital outcome after coronary artery bypass graft surgery. Am Heart J 2011;161:1179-85.e2.

8. Steinberg JS, Joshi S, Schron EB, et al. Psychosocial status predicts mortality in patients with life-threatening ventricular arrhythmias. Heart Rhythm 2008;5:361-5.

9. Spertus JA, Jones $P$, McDonell M, et al. Health status predicts long-term outcome in outpatients with coronary disease. Circulation 2002;106:43-9.

10. Ware J Jr, Kosinski M, Keller SD. A 12-Item Short-Form Health Survey: construction of scales and preliminary tests of reliability and validity. Med Care 1996;34:220-33.

11. De Smedt D, Clays E, Doyle F, et al. Validity and reliability of three commonly used quality of life measures in a large European population of coronary heart disease patients. Int J Cardio 2013;167:2294-9.

12. Zigmond AS, Snaith RP. The Hospital Anxiety and Depression Scale. Acta Psychiatr Scand 1983;67:361-70.

13. Bjelland I, Dahl AA, Haug TT, et al. The validity of the Hospital Anxiety and Depression Scale. An updated literature review. J Psychosom Res 2002;52:69-77.

14. Snaith RP. The Hospital Anxiety and Depression Scale. Health Qual Life Outcomes 2003;1:29.

15. Rabin R, de Charro F. EQ-5D: a measure of health status from the EuroQol group. Ann Med 2001;33:337-43.

16. Broadbent $\mathrm{E}$, Petrie $\mathrm{KJ}$, Main J, et al. The brief illness perception questionnaire. J Psychosom Res 2006;60:631-7.

17. Oldridge N, Hofer S, McGee HM, et al. The HeartQoL: Part I. Development of a new core health-related quality of life questionnaire for patients with ischemic heart disease. Eur J Prev Cardiol 2014;21:90-7.

18. Oldridge N, Hofer S, McGee HM, et al. The HeartQoL: Part II. Validation of a new core health-related quality of life questionnaire for patients with ischemic heart disease. Eur J Prev Cardiol 2014; 21:98-106.

19. Oldridge N, Saner H, McGee HM, et al. The Euro Cardio-QoL Project. An international study to develop a core heart disease health-related quality of life questionnaire, the HeartQoL. Eur $J$ Cardiovasc Prev Rehabil 2005;12:87-94.

20. Chang VT, Hwang SS, Feuerman M. Validation of the Edmonton Symptom Assessment Scale. Cancer 2000; 88:2164-71

21. Ezekowitz JA, Thai V, Hodnefield TS, et al. The correlation of standard heart failure assessment and palliative care questionnaires in a multidisciplinary heart failure clinic. J Pain Symptom Manage 2011;42:379-87.

22. Thygesen LC, Daasnes C, Thaulow I, et al. Introduction to Danish (nationwide) registers on health and social issues: structure, access, legislation, and archiving. Scand J Public Health 2011;

39(7 Suppl):12-6.

23. Pedersen CB. The Danish Civil Registration System. Scand J Public Health 2011;39(7 Suppl):22-5.

24. Lynge E, Sandegaard JL, Rebolj M. The Danish National Patient Register. Scand J Public Health 2011;39(7 Suppl):30-3.

25. Andersen JS, Olivarius Nde F, Krasnik A. The Danish National Health Service Register. Scand J Public Health 2011; 39(7 Suppl):34-7.

26. Petersson F, Baadsgaard M, Thygesen LC. Danish registers on personal labour market affiliation. Scand J Public Health 2011;39(7 Suppl):95-8.

27. Jensen VM, Rasmussen AW. Danish Education Registers. Scand J Public Health 2011;39(7 Suppl):91-4.

28. Baadsgaard M, Quitzau J. Danish registers on personal income and transfer payments. Scand J Public Health 2011; 39(7 Suppl):103-5.

29. Christensen Al, Ekholm O, Glumer C, et al. The Danish National Health Survey 2010. Study design and respondent characteristics. Scand J Public Health 2012;40:391-7.

30. Ekholm O, Gundgaard J, Rasmussen NK, et al. The effect of health, socio-economic position, and mode of data collection on non-response in health interview surveys. Scand J Public Health 2010;38:699-706. 